\title{
Late Motor Processing in Low-Impedance Robots: Impedance Control of Series-Elastic Actuators
}

\author{
Gill A. Pratt, Pace Willisson, Clive Bolton, and Andreas Hofman
}

\begin{abstract}
We have been working on several control and actuation improvements applicable to the design of biomimetic robots and assistive (e.g. prosthetic or orthotic) devices. This paper focuses on the implementation of a jointlevel impedance controller for series-elastic actuators that eliminates the use of joint angle sensor information, instead using information from co-located commutation sensors on the back of a brushless motor and a compression sensor on the series elasticity. This approach is both more robust than previous systems and less subject to instabilities due to stiction and backlash.
\end{abstract}

\section{INTRODUCTION}

Areas of the brain and spinal cord perform "late motor" processing that combines higher level commands with joint and muscle proprioception to control individual muscles[1]. We believe that late motor processing can also play an important role in enabling biomimetic robots and assistive devices to utilize simpler, lower-bandwidth high-level control. In this paper, we describe some initial steps we have taken to develop such a system, with joint actuators commanded by three parallel impedance parameters instead of one force parameter.

\section{LOW IMPEDANCE ACTUATION AND CONTROL}

Most robotic actuators are designed to have high (force/motion) mechanical impedance, i.e. to be "stiff". Despite the fact that animals typically have low mechanical impedance[2], many biomimetic robots built to date, such as the bipedal robots developed by Sony and Honda[3, 4], have control schemes whose output is a stiff motion trajectory, even though the stability parameter being controlled is fundamentally based on forces and torques.

An alternative approach, which we have explored in several past walking robots and assistive devices, is to utilize low-impedance "series-elastic" actuators [5, 6] and a low-impedance control language like "Virtual Model

Manuscript received March 17, 2004. This work was supported in part by the National Science Foundation under Grant \# 9733740.

G. A. Pratt is with the Franklin W. Olin College of Engineering, Needham, MA 02492. (e-mail: gill.pratt@olin.edu)

Pace Willisson and Clive Bolton are consultants to this project.

Andreas Hofman is with the MIT Computer Science and Artificial Intelligence Laboratory, Cambridge, MA 02139.
Control"[7]. Series-Elastic Actuators are composed of traditional high-impedance geared actuators coupled to loads via springs whose compression is measured and used to actively control the traditional part of the actuators. Series-elastic Actuators and Virtual Model Control have been successfully used to enable real 2-D robots to walk blindly over rough terrain at speeds limited only by mechanical power, and to enable simulated robots to accomplish terrain adaptation and natural-looking gait in 3$\mathrm{D}[8,9]$.

\section{IMPEDANCE CONTROLLED JOINT ACTUATION}

We are continuing to develop a low-impedance 3-D biped robot "M2"[10] and a variety of active low impedance assistive devices. During this work, we have come to realize that biomimetic late motor processing, in particular joint impedance control, has advantages over previous methods. In particular, we believe it makes little sense to build a high-gain force control loop in the actuator, trying to get as close to zero impedance as possible and then command the actuator from a higher level to create higher mechanical impedances.

In our new system, we inform the low-level actuator control system about the target impedance using the following interface:

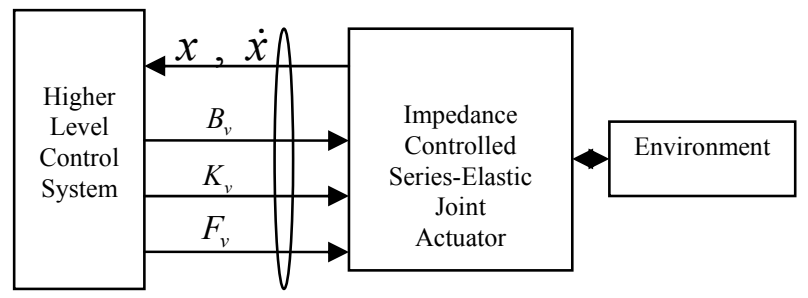

Figure 1: Late Motor Processing System Interface (circled)

Three parameters, $B_{v}, K_{v}$ and $F_{v}$, are sent from the higher level control system to the actuator control system. $B_{v}$ and $K_{v}$ command the actuator to create a virtual mechanical impedance equivalent to a parallel combination of a damper and a spring, respectively. $F_{v}$ commands the actuator to add an offset force in parallel with the damper 
and spring, or, when divided by $K_{v}$, an offset position in series with the virtual spring. As shown below, these are Norton and Thevenin Equivalents:
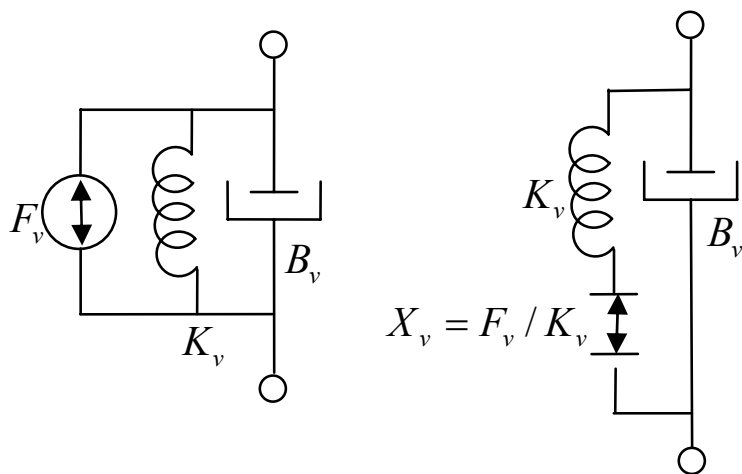

Figure 2: Norton and Thevenin Equivalents of Virtual Impedance

A single parameter $x$ and its derivative $\dot{x}$ are sent back to the higher level control system to give an indication of joint position and velocity. As will be explained later, these signals may be of low resolution.

\section{Series-Elastic Actuator Control}

A series-elastic actuator is composed of a traditional motor, a gear train, and a series-elasticity between the motor and the load:

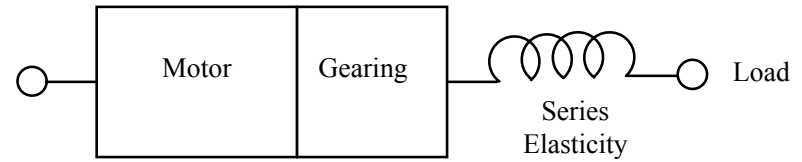

Figure 3: Schematic Diagram of a Series-Elastic Actuator

A photograph of the actuator used in this paper is shown below:

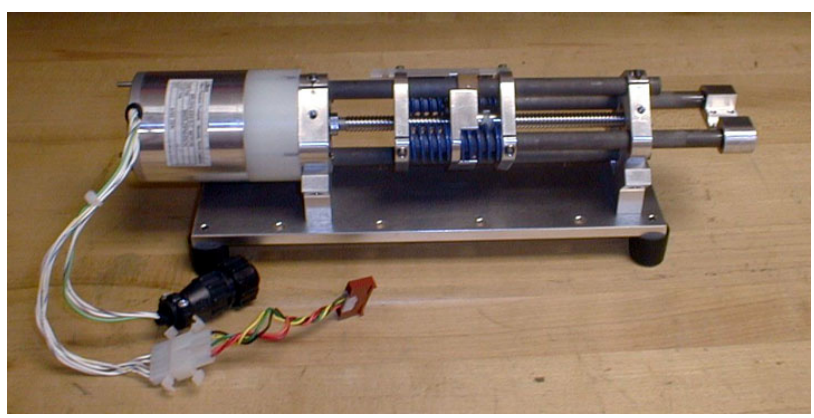

Figure 4: Photograph of Series-Elastic Actuator

The following system diagram can be used to represent the actuator plant:

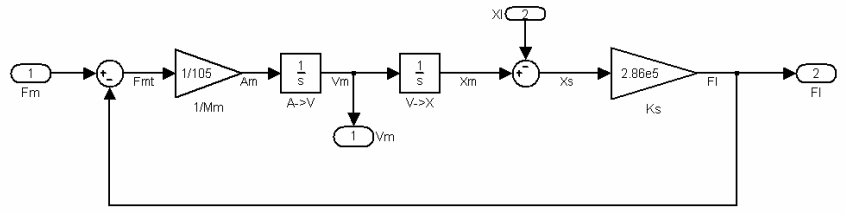

Figure 5: System Model of Actuator Plant

This model is referenced to linear co-ordinates. A force of $F_{m}$ Newtons due to motor current, along with the negative of the load force $F_{l}$ due to Newton's third law, creates a total force $F_{m t}$ that acts on the effective inertia of the motor's rotor $M_{m}=105 \mathrm{~kg}$ (this large linear inertia is due to the effect of the ball screw's pitch). The motor's rotor thus accelerates at $A_{m}=\frac{F_{m}}{M_{m}} \frac{m}{s^{2}}$, to a velocity $V_{m}=\int A_{m} d t$, and to a position of $X_{m}=\int V_{m} d t$. The difference between the motor's position $X_{m}$ and the load's position $X_{l}$ creates a compression in the series elasticity of $X_{s}=X_{m}-X_{l}$. This compression is converted by the elasticity's spring constant $K_{s}=2.86 \times 10^{5} \frac{\mathrm{N}}{\mathrm{m}}$ into a load force $F_{l}=K_{s} X_{s}$ (note that $X_{s}$ increases for increasing $X_{m}$ and decreases for increasing $X_{l}$ ).

In previous work[6] we created a force-controlled actuator by utilizing a current-controlled motor amplifier, and a high-gain feedback system controlling the commanded motor current (and thus the motor force $F_{m}$ ) based on the difference between a desired load force $F_{d}$ and the actual load force $F_{l}$. Some feed-forward terms were also added to improve performance:

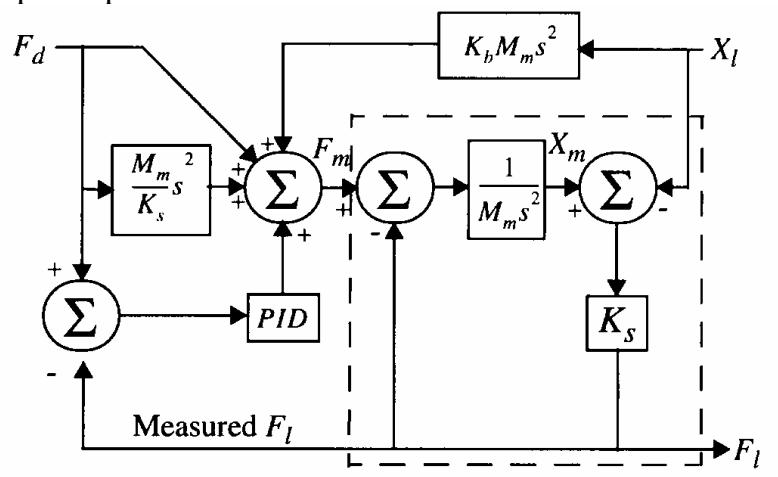

Figure 6: Previous Force Control System (model of actuator in dashed lines) 
The force-controlled actuator was connected around a revolute robot joint that included a joint angle sensor. The joint angle was fed to a higher level control system, which then (using Virtual Model Control) modulated the desired actuator load force $F_{d}$ in response to joint movements. This system, while workable, suffered from several drawbacks:

1. The non-collocation of the actuator and joint position sensor created instability (and thus limited the workable performance envelope) due to backlash in the attachment mechanism. This problem was particularly severe if large damping was desired at the joint.

2. Gear stiction was not sensed directly, as no motor position or velocity sensors were employed. Consequently, when stiction occurred the force feedback loop tended to respond slowly, then overshoot once stiction was overcome.

3. Computational load was high, as all actuators needed to be fed highly varying force commands at high sampling rates (in our case, every millisecond).

The first robot in which these drawbacks were partially addressed was the robot "Troody"[11], a life-size model of the dinosaur Troodon. As shown below, Troody used lowprecision spur gears between its brushed DC motors and the cable pulleys going to its series springs. Gear stiction and brush stiction forces were high. As a result, the force control was not smooth.

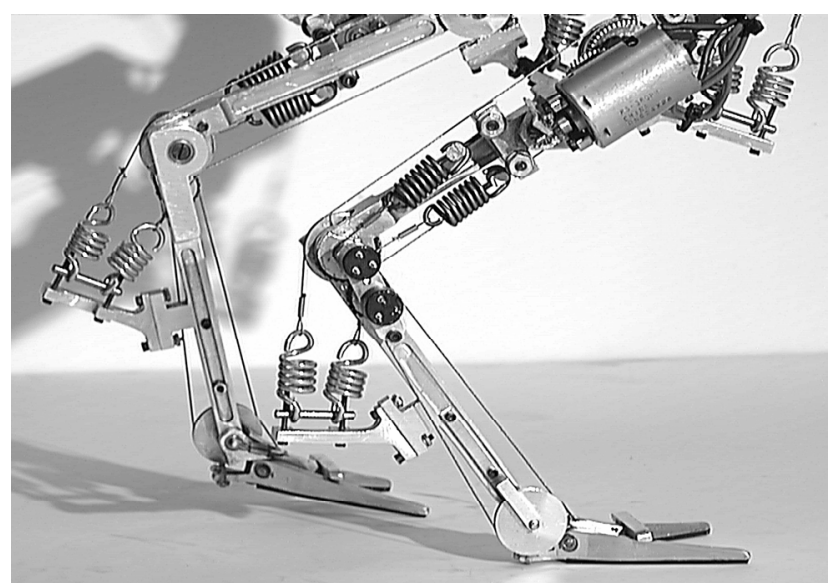

Figure 7: An early prototype of Troody

It was noticed that motor motion was much smoother when driven from a voltage rather than a current source, and thus a custom motor amplifier was constructed with an outer voltage loop, shown below:

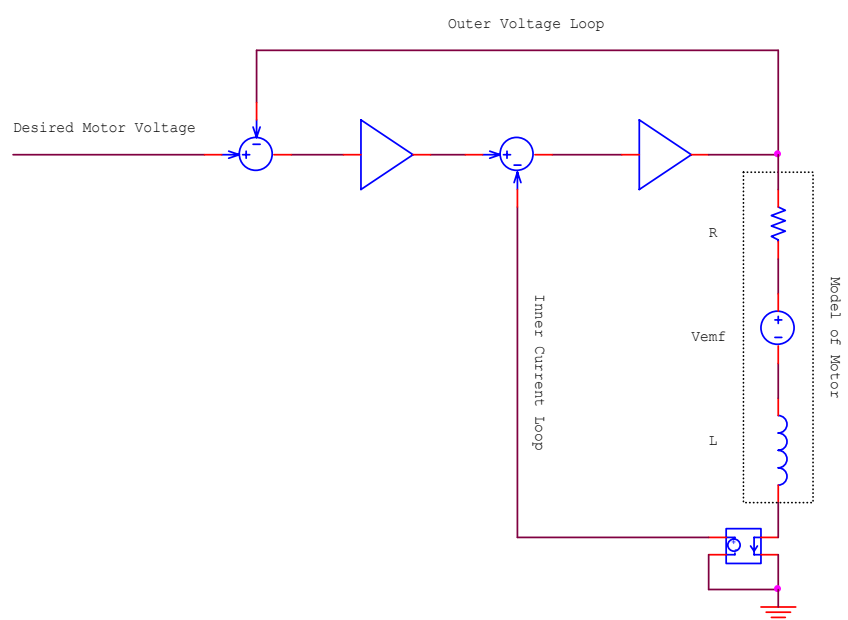

Figure 8: System Diagram of Inner Current, Outer Voltage Loop Motor Control. Each gain is a PI controller; last gain is Class D (PWM)

The desired motor voltage, rather than the desired motor current, was commanded based on the force error. An inner current control loop was still necessary due to the short time budget between unintended current overload and amplifier transistor destruction. However, this control loop operated at high bandwidth (several $\mathrm{KHz}$ ) and thus allowed for an outer loop controlling motor voltage. Ignoring the motor's inductance (since L/R was small), the motor voltage reflected the sum of the back-emf (itself a function of the motor's speed) and the IR drop of the windings. Thus, by controlling the motor's total voltage, a moderately high-gain velocity control loop was formed, capable of sensing motor and gear stiction and reacting to it very quickly with increased current, without the need for the slower force control loop to react (and then overshoot when the stiction had passed). The velocity loop was extremely stable, since the velocity sensors (the motor windings) and torque generating elements (the motor windings) were not only co-located, they were one and the same! The use of the motor as a bidirectional transducer between the electrical and mechanical realms was the key idea. To make the velocity loop even "stiffer", IR drop compensation could be used (although one must be careful to use the lowest possible compensating resistance over the actuator's temperature range to avoid instability). Troody's motor amplifiers were very dense, but "back EMF" based velocity controlled amplifiers are commercially available for both DC and Brushless motors (e.g. from Copley Controls[12]).

With voltage mode drive, Troody's motors "pushed through" brush and gear stiction easily by quickly raising and lowering motor current as needed with little impact on the slower force control loop. Troody's force control was smoother as a result. But Troody's joint sensors were still not co-located with its actuators, and the overall command interface to the actuators was still that of force control. 


\section{Eliminating JoINT ANGLE SENSORS}

For the following reasons, we currently believe that most Biomimetic robots and assistive devices do not need high resolution joint angle sensors:

1. We hypothesize that most balance and interaction tasks are based more on force than position, thus on-board inertial sensing and high fidelity force sensors for environment interaction may obviate the need for high resolution position sensors.

2. Humans typically have low joint position accuracy when doing tasks blindly (try closing your eyes and pointing to the upper right hand corner of this paper).

3. Clothing (e.g. shoes) typically inserts a significant disturbance to the location of our interaction with the environment. Yet, we can accomplish many tasks (like walking) despite such varying offsets. Equivalently, we can handle a certain amount of rough terrain (i.e. contact location disturbance) during locomotion even with our eyes closed.

4. Non-collocation of sensors and actuators is wellknown to exaggerate noise, hysteretic hunting, and large signal saturation instability particularly when damping impedances are desired. As opposed to haptic devices, where virtual damping must have high fidelity, damping in biomimetic robots and assistive devices is typically used to absorb gross motion energy from mass + spring (or pendulum) resonances, and the accuracy of the damping is less important than its guaranteed passivity.

5. Joint position sensors tend to be easily damaged, and hard to seal against the environment.

Thus, we hypothesize that impedance implemented at the collocated motor, motor shaft encoder, and spring compression sensor is sufficiently accurate for our purposes, and information from a joint position sensor is not required. In the case of the actuators in figure 4, a brushless motor provides us with a collocated encoder for free: the motor's commutation Hall-effect sensors. This particular motor (a Litton Poly-SCI BN23PM-03) has 8 poles, or 4 electrical cycles per mechanical revolution. With 6 commutation steps per electrical cycle, this gives us 24 encoder counts per revolution. The ball screw that was used has a pitch of $2 \mathrm{~mm}$ per revolution, yielding an effective overall linear resolution of $82 \mu \mathrm{m}$ per motor hall sensor step. To utilize this information, we constructed an FPGA based interface board that "spies" on the hall-effect signals as they go from the brushless motor's sensors to the motor amplifier. A photograph of this interface board, for half of the robot's 12 degrees of freedom, is shown below. Besides tapping the hall-effect signals, this board also contains decoders and counters for the hall sensors, and a large number of $\mathrm{D} / \mathrm{A}$ and $\mathrm{A} / \mathrm{D}$ converters with associated analog signal conditioning hardware. The board interfaces to the control computer through a fire-wire (IEEE 1394) interface running at $400 \mathrm{MBits} / \mathrm{s}$. This provides more than sufficient bandwidth for a $1 \mathrm{KHz}$ update rate with unit delay latency in the control loop.

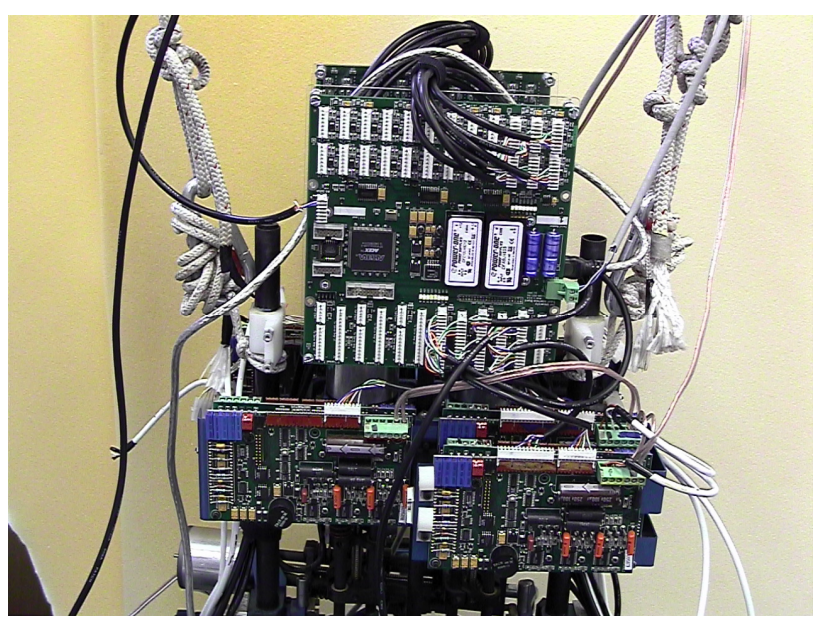

Figure 9: Photograph of interface board (top) and motor amplifiers (bottom)

\section{PROGRAMMEd IMPEDANCE CONTROL}

With only the actuator's hall sensors and spring compression sensor as inputs, we designed a simple control system that allowed us to create the desired virtual joint impedance. Unlike the situation with Troody, we did not have a measure of motor voltage, so back-EMF based velocity control could not be used. Instead, we employed a traditional high-gain position controller on the hall-based motor position $X_{m}$ :

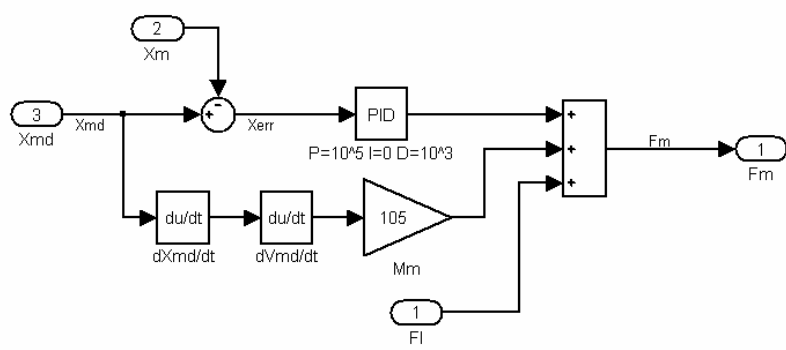

Figure 10: Motor Position Controller

A unity gain positive feedback path for $F_{l}$ was provided to cancel the Newton's $3^{\text {rd }}$-law force required to support the load force. This positive feedback eliminates, to first order, disturbances due to load motion, as any sensed load imposed externally immediately causes "push-back" by the 
motor. It also removes the effort required to compress the spring from the feedback loop. This positive feedback is marginally stable, but is stabilized by the negative feedback position loop. A feed-forward path is also provided to compensate for the inertia of the motor rotor, leaving the position feedback only canceling unmodeled dynamics.

We need to command the desired motor position $X_{m d}$ based on the sensed load force $F_{l}$. We know that a load force of $F_{l}$ will create a spring compression of:

$$
X_{s}=\frac{F_{l}}{K_{s}}
$$

This leads to a computed desired motor position $X_{m d}$ with respect to the desired load position $X_{l}$ of:

$$
X_{m d}=X_{l}+X_{s}=X_{l}+\frac{F_{l}}{K_{s}}
$$

or, in terms of $X_{l}$ :

$$
X_{l}=X_{m d}-\frac{F_{l}}{K_{s}}
$$

To implement the desired virtual impedance dictated by $F_{v}, B_{v}$, and $K_{v}$, we wish for the load force to obey the relationship:

i.e.:

$$
F_{l}=F_{v}-K_{v} X_{l}-B_{v} \dot{X}_{l}
$$

$$
F_{l}=F_{v}-K_{v} X_{m d}+\frac{K_{v}}{K_{s}} F_{l}-B_{v} \dot{X}_{m d}+\frac{B_{v}}{K_{s}} \dot{F}_{l}
$$

In our implementation, $K_{s}=2.86 \times 10^{5}$ which is significantly higher than the virtual damping $B_{v}$ we wish to create. This makes the final term in the above equation a small contributor to the overall control law if damping at Biomimetic frequencies (a few $\mathrm{Hz}$ ) is desired. This term also contributes to noise (due to the derivative taking of the force sensor signal). Leaving it out effectively rolls off the response of the damping beyond frequencies of $\frac{K_{s}}{B_{v}}-\mathrm{a}$

desirable effect. Thus, we ignore the final term in the above equation, arriving at:

$$
F_{l}=F_{v}-K_{v} X_{m d}+\frac{K_{v}}{K_{s}} F_{l}-B_{v} \dot{X}_{m d}
$$

If we have a velocity or motor voltage controller (such as was used in Troody), we can prevent drift by substituting the actual motor position $X_{m}$ for $X_{m d}$ and solve for $\dot{X}_{m d}$ :

$$
\dot{X}_{m d}=\frac{F_{v}-K_{v} X_{m}+\frac{\left(K_{v}-K_{s}\right)}{K_{s}} F_{l}}{B_{v}}
$$

This will work well as long as $B_{v}$ is not too small - an easy to achieve result by setting a reasonable minimum (e.g. $\left.B_{v}=10 \frac{N s}{m}\right)$. The actuator we use can generate roughly $1700 \mathrm{~N}$, so at a typical speed of $\dot{X}_{m}=1 \frac{\mathrm{m}}{\mathrm{s}}$, we will incur a minimum "drag" of only $10 N$, which is on the order of the force sensor's resolution.

In the case of our new robot, without motor voltage control, we require a solution for the desired motor position $X_{m d}$ :

$$
X_{m d}=\frac{F_{v}+\frac{\left(K_{v}-K_{s}\right)}{K_{s}} F_{l}-B_{v} \dot{X}_{m d}}{K_{v}}
$$

In continuous time control, this could be implemented by integrating both sides of this equation and following the resulting control law.

Our discrete time controller (which uses simple Euler approximations to derivatives), allows us to write:

$$
X_{m d[t]}=\frac{F_{v}+\frac{\left(K_{v}-K_{s}\right)}{K_{s}} F_{l[t]}-B_{v} \frac{\left(X_{m d[t]}-X_{m d[t-1]}\right)}{\Delta t}}{K_{v}}
$$

which reduces to:

$$
X_{m d[t]}=\frac{F_{v}+\frac{\left(K_{v}-K_{s}\right)}{K_{s}} F_{l[t]}+\frac{B_{v}}{\Delta t} X_{m d[t-1]}}{K_{v}-\frac{B_{v}}{\Delta t}}
$$

Note that in this case, either $K_{v}$ or $B_{v}$ should be above some minimum in order to avoid excessive amplitudes of $X_{m d}$.

\section{EXPERIMENTAL RESULTS}

After simulations were performed, an isolated actuator (on the ankle) of the M2 robot was driven with the new control system. A number of virtual impedances of varying amplitude were commanded, three of which are reproduced below. In the first figure, the motor was commanded to act like a spring so that $\frac{K_{v}-K_{s}}{K_{s}}=10,000 \mathrm{~N} / \mathrm{m}$. Traces of the system's operation for a hand-applied disturbance are 
shown below. Note that the motor's displacement corresponds to the load force:

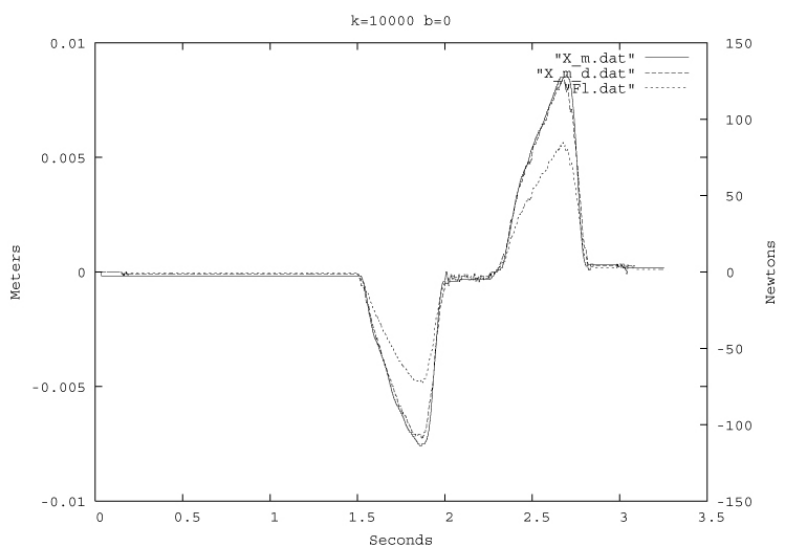

Figure 11: Operation as a virtual spring (first $0.25 \mathrm{~s}$ contains a calibration artifact)

The following figure shows operation with $B_{v}=10,000 \mathrm{Ns} / \mathrm{m}$. Note that the motor position follows the integral of the force, as expected:

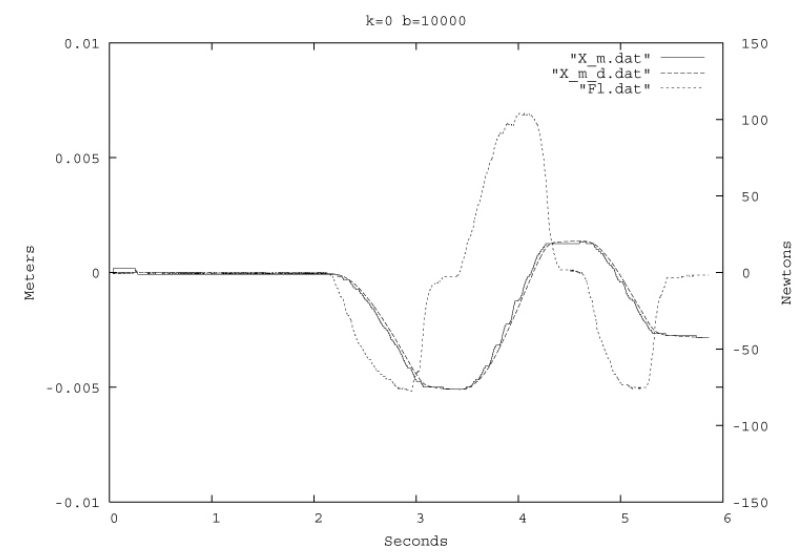

Figure 11: Operation as a Virtual Damper (first $0.25 \mathrm{~s}$ contains a calibration artifact)

The final figure below shows operation with both virtual motor damping and stiffness set to 10,000 . Note how the spring/damper combination recovers with exponential decay:

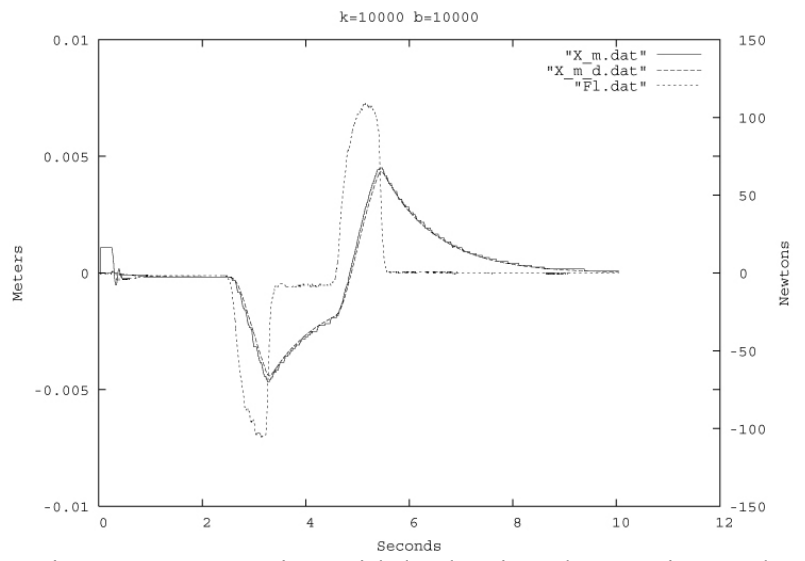

Figure 12: Operation with both Virtual Damping and Virtual Stiffness

(first $0.25 \mathrm{~s}$ contians a calibration artifact)

\section{CONCLUSIONS}

We have shown how a simple impedance control system can be constructed to drive a series-elastic actuator, with Damping, Stiffness, and Force Offset as the command inputs. The control system provides the following benefits:

1. It does not require a load position sensor, but still generates impedances that we believe are sufficiently high fidelity for Biomimetic tasks.

2. It has high motor position / velocity gain, stably rejecting force disturbances such as stiction.

3. It uses only as much force-sensor gain as is required for the desired impedance, increasing stability margins and lowering noise.

4. It may free the higher level control system to operate at a slower control rate, since we believe that Damping, Stiffness, and Offset vary more slowly than resultant actuators forces for Biomimetic tasks. This is the subject of ongoing work.

\section{REFERENCES}

S. F. Giszter, F. A. Mussa-IVAldi, AND E. BizzA, "CONVERGENT FORCE FIELDS ORGANIZED IN THE FroG'S SimAl CORD," THE JOURNAL OF NEUROSCIENCE, VOL. 12, PP. 467-491, 1993.

C. T. FARLEY, J. GLASHEEn, AND T. A. MCMAHON, "RUNNING SPRINGS: SPEED AND ANIMAL SIZE," JOURNAL OF EXPERIMENTAL BIOLOGY, VOL. 185, PP. 71-87, 1993. TAKAHASHI, AND T. MATSUMOTO, "LEGGED 
MOBILE RoBot EQUIPPED WITH IMPACT

ABSORBER." US PATENT 5,445,235: HONDA GIKEN

KOGYO KABUSHIKI, 1995.

[4] K. Hirai, M. HiRose, Y. HaikaWA, AND T. TAKENAKA, "THE DEVELOPMENT OF HONDA HUMANOID ROBOT," PRESENTED AT IEEE INTERNATIONAL CONFERENCE ON ROBOTICS AND Automation, 1998.

[5] G. A. Pratt and M. M. Williamson, "StifFness ISN'T EVERYTHING," PRESENTED AT FOURTH INTERNATIONAL SYMPOSIUM ON EXPERIMENTAL RовотICS, 1995.

[6] G. A. Pratt and M. M. Williamson, "Series ELASTIC ACTUATORS," PRESENTED AT IEEE INTERNATIONAL CONFERENCE ON INTELLIGENT ROBOTS AND SYSTEMS, 1995.

[7] J. Pratt, P. Dilworth, AND G. Pratt, "Virtual MODEL CONTROL OF A BiPEDAL WALKING ROBOT," PRESENTED AT IEEE INTERNATIONAL CONFERENCE ON ROBOTICS AND AUTOMATION, 1997.

[8] J. Pratt, A. Torres, P. DilWorth, and G. Pratt, "Virtual ACtuator Control," PRESENTED AT IEEE INTERNATIONAL CONFERENCE ON INTELLIGENT RoBOTS AND SYSTEMS, 1996.

[9] C.-M. Chew, J. Pratt, AND G. Pratt, "Blind WALKING OF A PLANAR BIPEDAL ROBOT ON SLOPED TERRAIN," PRESENTED AT IEEE INTERNATIONAL CONFERENCE ON ROBOTICS AND AUtOMATION, 1999.

[10] D. W. Robinson, J. E. PRATt, D. J. PALUSKa, AND G. A. Pratt, "SERIES Elastic Acutator DEVELOPMENT FOR A BIOMIMETIC ROBOT," PRESENTED AT IEEE/ASME INTERNATIONAL CONFERENCE ON ADVANCED INTELLIGENT MECHATRONICS, 1999.

[11] P. DilWORTH, "HTTP://WWW.AI.MIT.EDU/PEOPLE/CHUNKS/CHUNK S.HTML."

[12] Copley Controls, "HTTP://WWW.COPLEYCONTROLS.COM." 